an ointment composed of one drachm of the tartarized antimony and an ounce of lard, which was continued until a large crop of pimples was brought out. I dressed the stump every day with pledgets of lint dipped in a lotion of the chloruret of soda (and occasionally Bates's wash), simple dressing, compress of lint, strips of adhesive plaster, and a bandage. The ancle continuing to get worse and more painful, I determined upon the employment of iodine externally, having used it successfully in similar cases, and accordingly ordered it to be used, night and morning, combined with the extract of belladonna. Under this treatment the ancle began to improve, and in the space of a fortnight from this application, the ancle was quite well, except contraction of the joint, which was afterwards restored by the use of moxa; I now applied poultices to the arm, and ordered him medicines to better his general health, and to rub in indine over the abdomen, a practice which I have pursued in numerous cases with the greatest success. I also made an issue in his arm, having in several cases witnessed the ill effects of entirely removing longcontinued discharges from the system. Under this treatment his health rapidly improved, and in five months his stump was perfectly healed. In January 1829 , I removed a piece of bone from the abscess which had formed upon his arm, and he was in perfect health, except as regarded the healing of the abscess, which was impeded by another portion of bone, which 1 removed on the 12th of March 1829. This healed up in two or three days.

Since the above-mentioned time, now a period of four years, he has not had any return of his complaint, and, instead of being a poor emaciated creature, is a stout healthy man, pursuing his avocations with comfort and pleasure.

\section{CONTRACTION OF THE UTERUS.-ERGOT} OF RYE.

\section{To the Editor of The LANCET.}

Sir,-In No. 490 of The Lancet (January $19 \mathrm{th}$ ), there is the report of a debate on the function of the uterus, which occurred at the London Medical Society, and in which a variety of discordant opinions was expressed on the powers of that organ. It appears somewhat extraordinary that so much difference of sentiment should exist amongst well-informed men, - who have had extensive opportunities of observing the admirable and almost omnipotent powers of the uterus in the process of parturition.
In the debate in question one gentleman states, or implies, that with almost the touch of a finger he can retard the labour, to suit his convenience, or ensure the safety of the perineum; while another, equally positively, states the power to be strong enough to overcome all resistance, even "to eject him from the bedside of the patient, or out of the room." Do these differences arise from want of close observation, or from the ever-varying power of the uterus itself? However it be, the young practitioner must be sadly perplexed, when he comes to the bed-side of the patient, to know how to act.

Hour-glass contraction of the uterus (the subject which gave rise to the discussion) is, I consider, of rather rare occurrence, for in about 1500 cases that $I$ have attended, I find, on reference to my note-book, but two of hour-glass contraction. No mention is made of hasty delivery; but it does not unfrequently happen in wellformed women, that the child is expelled after the rupture of the membranes, by one long-continued effort, which no force that it is prudent to employ can for a moment prevent.

The contraction of the os uteri, enclosing the placenta, is of more common occurrence, but is easily overcome by carrying the fingers along the funis, and insinuating them, wedge-like, through the contraction. Some hemorrhage occasionally occurs, but, as far as my observation goes, not to an alarming extent.

The regular contraction of the uterus is secured and much facilitated by grasping it with the hand externally, immediately after the expulsion of the foetus - a practice I invariably adopt; and, unless there is some preternatural adhesion of the placenta, by gently drawing the chord with the right-hand, while the uterus is grasped with the left, it may be generally withdrawn, and the uterus securely contracted within ten minutes after the birth of the child, when alarming hemorrhage will rarely be found to follow. The placenta will sometimes continue for an hour or two in the womb, that organ being in a state of quiescence during the time. I have sometimes witnessed this in cases attended by my pupils; but the moment the uterus is grasped, rather firmly, by the hand externally, it may be felt to contract forcibly, and the placenta is immediately expelled by the means stated before.

When such a diversity of opinion is entertained as to the natural powers of the uterus,- a power much influenced by the constitutional irritability of the patient,we need not be surprised at the various statements respecting the powers of the secale cornutum. Some persons, judging 
from the results of a solitary case or two, imagine it only necessary to be armed with a drachm or so of this potent remedy, when all difficulties must vanish. The vectis, forceps, and even the perforator, are no longer required; but, like the wishingcap of the Arabian Knights, it subdues all obstacles, and makes everything bend to the will of its possessor. Now without denying the specific effects of the ergot upon the action of the uterus, I think we ought to be extremely cautious in giving a positive opinion on a subject, when, from the nature of the circumstances, we can have but negative evidence; especially with such a capricious organ as the one in question. It is well known, that after a woman has had trifling and inefficient pains for hours, violent and long-continued action will come on suddenly, and without any obvious reason. Also, from protracted and ineffectual efforts, when there is rigidity of the soft parts, or from other causes, the head of the patient will become confused, the face fiushed, and the pulse full and slow, from local congestions. Here, after relieving the system by the abstraction of from 12 to 20 ounces of blood, the energies of nature will be restored, and the delivery accomplished without medicinal aid. Add to this, when two or three half-drachm doses of the ergot are given, as I have very frequently done, without any, or very little, increased action of the uterus, it is not unreasonable to doubt the infallible power which has been ascribed to it by some writers who have occupied the pages of your valuable journal. For it ought to be remembered, that when the ergot is given without effect, the evidence is positive of its failure; while, on the contrary, we can never be certain that the increased action following the administration of the medicine arises from its effects.

I am, Sir,

Your very obedient servant, Thomas Weddell.

Scarborough, March 15th, 1833.

Lithotrity in America.-Though we have not, in America, yet enjoyed the advantage of witnessing the application of the new instruments by professed lithotritists, the few successful trials made by our own surgeons, some of whom have practised in France under Civiale and Le Roy, aided by the mass of testimonies in its favour from abroad, have in great measure silenced the opposition of those who used to sneer at it as a piece of French humbug. The British surgeons seem now generally to regard lithotrity as a highly superior operation.-Amer.Journ. Med.Sci.
WESTMINSTER MEDICAL SOCIETY;

Saturday, March 30th, 1833.

\author{
Dr. Jewee in the Chair.
}

FUNCTIONS OF THE RECTUM.

[At the meeting of April 6th, a man is to be exhibited by Mr. Holt, , the surface of whose body, excepting the face, palms, and soles, presents rather the character of a beast's hide, than a human skin,--an unique disease allied to icthyosis or elephantiasis, in appearance, and in some parts bristly with hair.] This evening,

Mr. North requested that the anatomists present would give their opinion on the theory of Dr. O'Beirne, who, in a work on "Defecation," lately published, contends that the rectum is not, as usually taught by anatomists, a " receptacle for fæces," but a kind of sphincter intestinorum, - whose office is to the whole alimentary canal, that which the sphincters ani are usually considered to be to the rectum.The question (observed the speaker) involves an important practical point, for it tends to account for the frequent inefficiency of enemata, and to show that (as Dr. O'B. recommends). in order to secure their effectual administration, a tube should first be introduced up to the termination of the sigmoid flexure of the colon, the point at which, he says, the canal ceases to contain frees, except at the moment before an evacuation is bergun. Dr. O'Beirne believes that clysters, according to the ordinary mode of exhibition, only go as high as the termination of the colon, instead of being injected among the frees. His work is a very pertinent one, has the merit of originality, and contains views of great practical utility, and has been more tartly treated in one or two publications, than it seems to me to deserve. The course the author pursued was this: he first observed certain facts tending to establish his views, then apparently confirmed them by experiment, and next told them to his friends, who then put them to the test in cases of obstinate constipation, tympanitis, and produced confirmatory results. As I see several anatomists in the room, I have risen to ask if they are aware of the existence of any valve, or anything so very angular in the mode in which the sigmoid flexure of the colon terminates, as to be calculated to prevent the fæces from entering the rectum, except at the moment when an evacuation is about to take place.

* Sir Ándrew Cant's make-believe-holiness Bill not yet having the power to prevent medical secieties from profaning the Easier week. 